\title{
Identification of cave minerals by Raman spectroscopy: new technology for non-destructive analysis
}

\author{
William B. White ${ }^{1}$

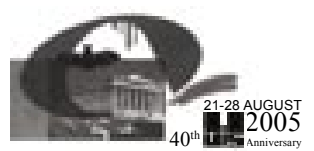 \\ Paper presented during the 14th International Congress of Speleology, Kalamos (Greece) 21-28 August 2005.
}

\begin{abstract}
:
White W. B. 2006. Identification of cave minerals by Raman spectroscopy: New technology for non-destructive analysis. International Journal of Speleology 35 (2), 103-107. Bologna (Italy). ISSN 0392-6672.

The identification of minerals from caves generally requires that samples be removed from the cave for analysis in the laboratory. The usual tools are X-ray powder diffraction, the optical microscope, and the scanning electron microscope. X-ray diffraction gives a definitive fingerprint by which the mineral can be identified by comparison with a catalog of reference patterns. However, samples must be ground to powder and unstable hydrated minerals may decompose before analysis is complete. Raman spectroscopy also provides a fingerprint useful for mineral identification but with the additional advantage that some a-priori interpretation of the spectra is possible (distinguishing carbonates from sulfates, for example). Because excitation of the spectra is by means of a laser beam, it is possible to measure the spectra of samples in sealed glass containers, thus preserving unstable samples. Because laser beams can be focused, spectra can be obtained from individual grains. New technology has reduced the size of the instrument and also the sensitivity of the optical system to vibration and transport so that a portable instrument has become possible. The sampling probe is linked to the spectrometer by optical fibers so that large specimens can be examined without damage. Comparative spectra of common cave minerals demonstrate the value of Raman spectra as an identification technique.
\end{abstract}

Keywords: Raman spectroscopy, cave minerals, calcite, aragonite, gypsum

Received 3 January 2006; Revised 13 March 2006; Accepted 12 June 2006

\section{INTRODUCTION}

The identification of minerals from caves poses some unique problems. Some minerals, such as calcite and gypsum, are common, occur as large crystal grains, and can often be identified from visual inspection. Others are fine-grained, nondescript powders that require instrumental analysis. Some cave minerals are not stable when removed from the cave. Their stability demands the cool, damp cave environment and they readily decompose into other compounds when brought into the warm, dry surface environment. For many delicate speleothems, specimens should not be removed from the cave at all, or if samples are removed, they become scientifically valuable specimens that should be analyzed in the least destructive way possible so that the specimens can be preserved for possible future study.

Beyond visual inspection and examination by binocular microscope, the analytical tool of choice is powder X-ray diffraction. X-ray powder patterns

1) Materials Research Institute and Department of Geosciences The Pennsylvania State University, University Park,

PA 16802 USA - e-mail: wbw2@psu.edu provide unique fingerprints that can be matched against catalogs of standards. X-ray powder diffraction has the drawback that samples must be ground to a fine powder for measurement. Grains, which may consist of different minerals, are mixed together. Possible decomposition in the ambient atmosphere is enhanced. A further limitation of X-ray powder diffraction is that there is no easily interpreted relationship between the diffraction lines and the chemical composition of the sample. The patterns can be calculated from the crystal structure of the mineral but the calculation proceeds from the known structure to the diffraction pattern, not the reverse. Other characterization tools such as the scanning electron microscope with energy dispersive X-ray spectroscopy and the electron microprobe have important uses but there remains a need for additional tools for cave mineral investigations.

The purpose of the present paper is to describe the application of Raman spectroscopy to cave mineral investigations and to point out certain special features that address some of the shortcomings described above. In particular, new technologies have been developed that make the application of Raman 
spectroscopy to cave mineralogy more tractable than it has been in the past.

\section{PRINCIPLES OF RAMAN SPECTROSCOPY}

Raman spectroscopy is an inelastic light scattering experiment. If a transparent specimen is illuminated by an intense, monochromatic light source such as a laser, light is scattered from the specimen in all directions. For imperfect specimens such as most minerals, most of the scattering will be due to flaws, inclusions, and other sources of cloudiness. If the sample is perfectly transparent, the intensity of the light scattered perpendicular to the beam will be extremely weak but not zero. Most of this weak scattered light, called Rayleigh scattering, will have the same wavelength as the incident laser beam. However, a very small fraction of the scattered light will interact with the specimen, set its molecular structure into vibration, and appear in the scattered light as extremely weak component with wavelengths shifted by certain discrete values from the laser wavelengths. The wavelength shifts are a measure of the vibrational frequencies of the chemical bonds in the specimen. This weak component is the Raman scattering. If the scattered light is passed through a monochromator to display the component wavelengths, the Raman scattered light appears as side bands on the much more intense central peak of the Rayleigh scattering (Fig 1). The usual convention is for Raman spectra to be

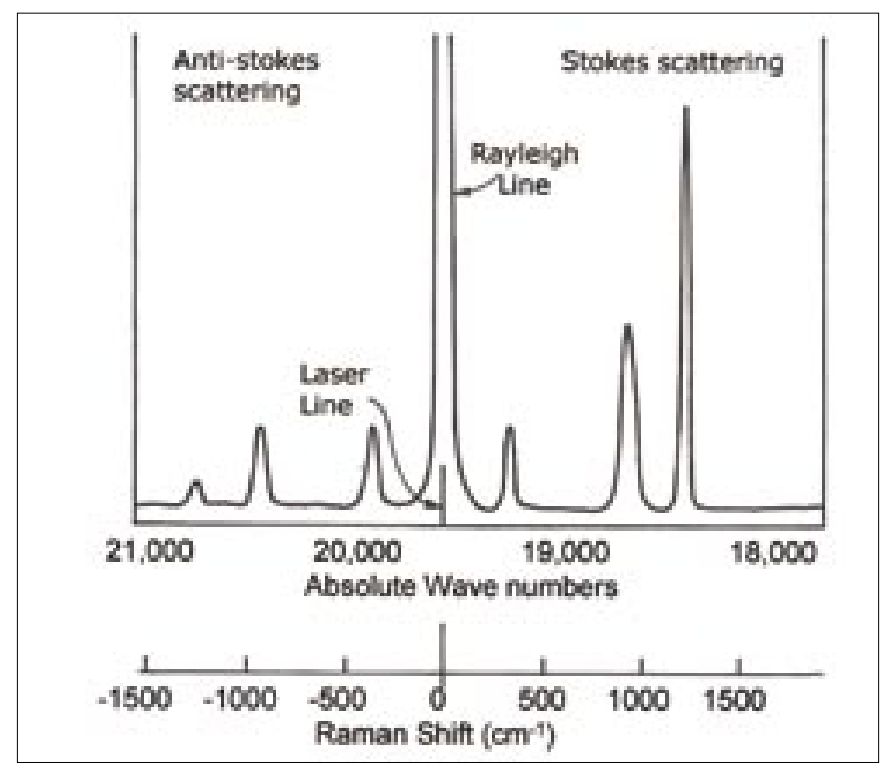

Fig. 1. Schematic Raman spectrum. Note that Raman lines appear at both higher and lower wavenumbers from the exciting (Rayleigh) line. Usually, only the low wavenumber side is displayed, with the wavenumber of the exciting line set equal to zero.

plotted in units of wavenumbers, $\mathrm{cm}^{-1}$, the inverse of the wavelength in units of centimeters measured from the wavenumber of the exciting laser line taken as zero. In these units, the wavenumbers of the Raman bands are proportional to the frequencies of the vibrations of the molecules or crystals.

Although the Raman effect was discovered in 1928 it did not at once produce a new analytical technique. The problem is that the Raman effect is extremely weak; Raman scattered intensities are on the order of $10^{-10}$ of the intensity of the exciting source. The Raman signal is easily lost in stray light and measurement on other than perfectly transparent samples was impossible. Two inventions were made in the 1960's: the laser as an ultra-intense excitation source and double (or triple) monochromator optics to extract the desired signal from the stray light background. With these inventions, spectra could be measured routinely on cloudy crystals, colored crystals, and even opaque crystals, exactly what was needed for the analysis of minerals. Many further extensions of the technology have been made (Adar, 2001), two of which are discussed in this paper.

The number, wavenumbers, and intensities of the Raman bands are determined by the symmetry and arrangement of the atoms within the unit cell, by their masses, and by the strength of the bonds that hold them together. Raman spectra, therefore, are closely related to infrared spectra which are another way of measuring vibrational frequencies. The physics of these interactions is complex but well known (Wilson, Decius and Cross, 1955; Long, 1977). For present discussion, the Raman spectrum can be considered a fingerprint with no need to be concerned with the underlying physics. Equipment suitable for general laboratory use appeared on the market in the early 1970's and it was quickly applied to problems in mineralogy (White, 1975). Because Raman spectra are measured by simply reflecting a laser beam from the sample, the technique is non-destructive and can be applied to samples of irregular size and shape. As a characterization tool, Raman spectroscopy has been applied to archaeological materials (Smith and Clark, 2004), gems (Kiefert, Hänni and Ostertag, 2001) and synthetic diamond films (Knight and White, 1989).

\section{MICROFOCUS RAMAN SPECTROSCOPY}

Because Raman spectra are measured with visible light lasers, it is possible to bring the exciting beam through the optics of an ordinary microscope and focus it on a specimen on the microscope stage. The beam interacts with the sample and then reflects back through the microscope where the beam is split from the incoming beam and sent to the monochromator and detector. Thus measurements can be made on individual crystal grains with the spatial resolution of the microscope - about $2 \mu \mathrm{m}$ (Fig. 2). For speleothems, this means that the mineralogy of multimineralic speleothems can be examined one grain at a time without damage to the specimen itself. Alternatively, one could prepare thin sections of speleothems for examination under the polarizing light microscope and also for grain-by-grain mineral identification by Raman spectroscopy.

Some stalactites in cross-section show alternating layers of calcite and aragonite. The appearance of both minerals in this relationship has important climatic implications but if the layering is on a fine 


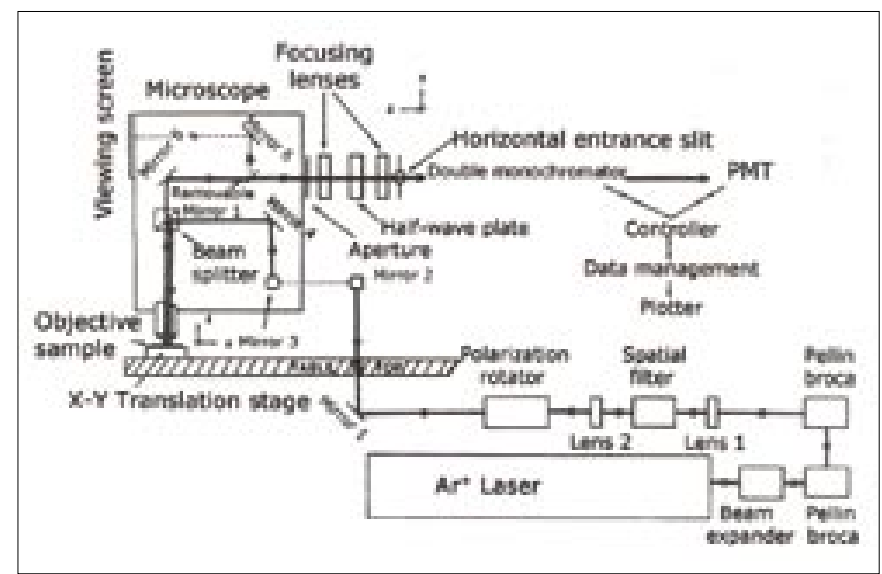

Fig. 2. The optical system of a microfocus Raman spectrometer. Only the laser steering optics and the beam-splitting arrangement within the microscope are shown. The double monochromator, the detector (PMT = photomultiplier tube), and computer system are only roughly indicated.

scale, layer-by-layer mineral identification is difficult. By placing a section of stalactite on the microscope stage, the laser beam can be focused on the individual layers and the component minerals identified.

The Raman spectra of calcite and aragonite are shown in Fig. 3. Note that the high wavenumber features are very similar but the low wavenumber features are different. The high wavenumber bands are the internal vibrations of the carbonate ion which is an essential component of both calcite and aragonite. The low wavenumber bands arise from vibrations of the ionic arrangement within the crystals so these reflect the quite different structures of calcite and aragonite and allow the identification of either mineral, or a mixture of both. A full theoretical analysis of the calcite and aragonite spectra is given by White (1974). It should be noted that Raman spectroscopy is not useful for distinguishing calcite from dolomite. These two minerals have, except for the ordering of $\mathrm{Ca}^{2+}$ and $\mathrm{Mg}^{2+}$ ions in dolomite, the same crystal structure and thus very similar spectra. The ordering reduces the space group from $R \overline{3} c$ for calcite to $R \overline{3}$ for dolomite. Theoretical analysis predicts a relaxation of some selection rules, but the predicted new bands have not been observed.

Because the samples to be measured are simply placed on a microscope stage, the microfocus Raman spectrometer allows the possibility of using a waterimmersion lens so that crystals can be measured immersed in an aqueous solution. Mirabilite, $\mathrm{Na}_{2} \mathrm{SO}_{4} \cdot 10 \mathrm{H}_{2} \mathrm{O}$, is a commonly occurring sulfate mineral. It is stable in a saturated solution but unstable in the ambient surface atmosphere where it decomposes to anhydrous $\mathrm{Na}_{2} \mathrm{SO}_{4}$, the mineral thenardite. The decomposition is very rapid so that it is difficult to identify mirabilite in speleothems because it decomposes while X-ray diffraction patterns are being measured. With the water immersion lens, it was possible to obtain a good quality spectrum of mirabilite immersed in its own saturated solution (Fig. 4).

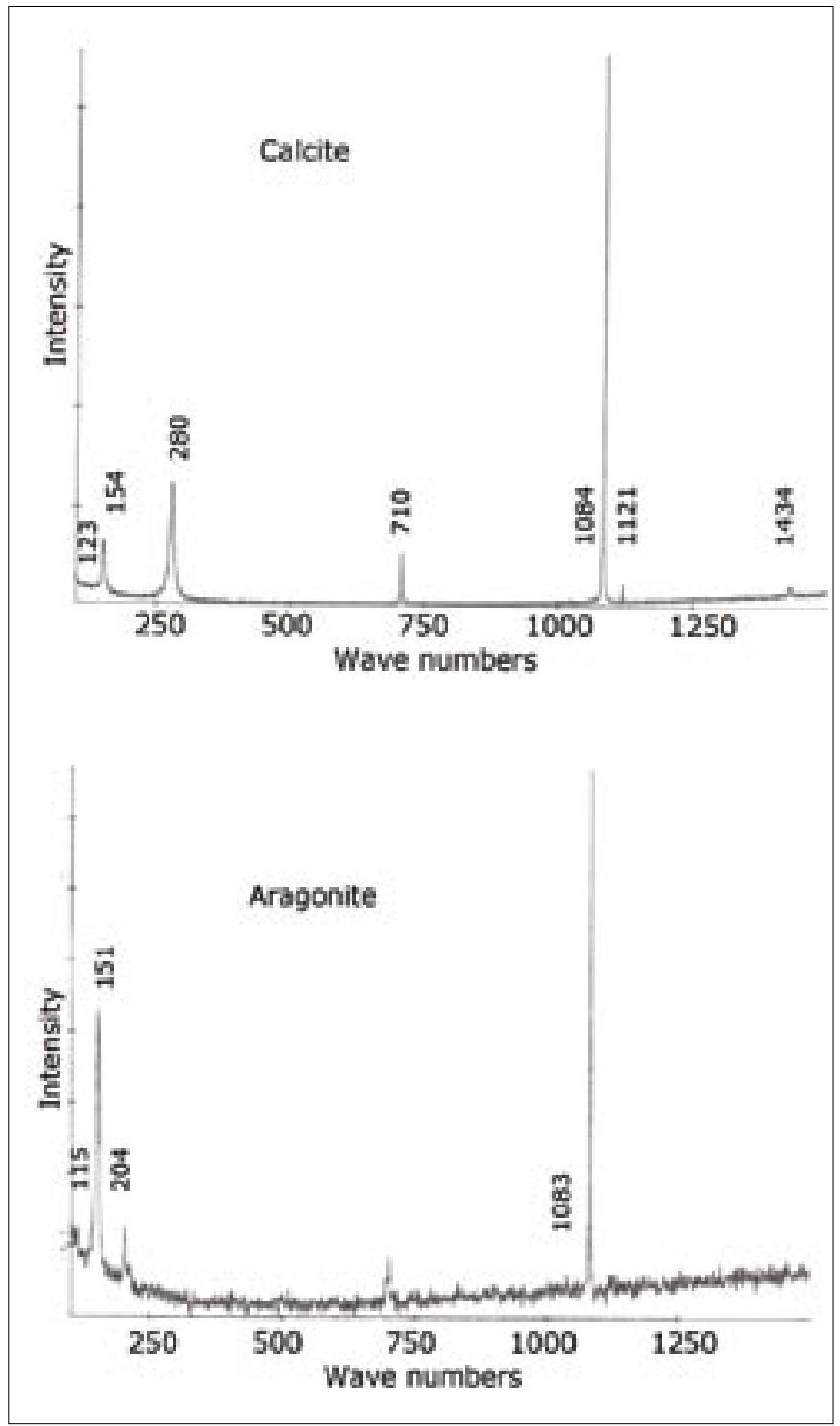

Fig. 3. Raman spectra of calcite and aragonite.

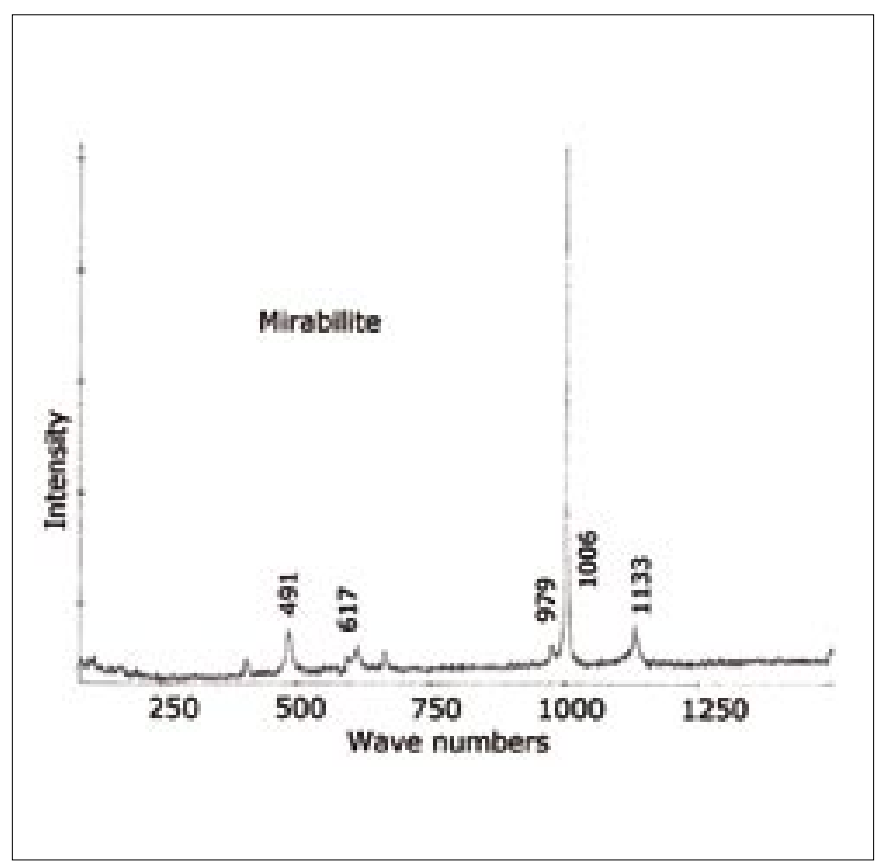

Fig. 4. Raman spectrum of mirabilite crystal in $\mathrm{Na}_{2} \mathrm{SO}_{4}$-saturated water. 


\section{PORTABLE FIBER OPTIC SPECTROMETERS}

The most recent improvement in technology, and the one that inspired the present paper, is the fiber optic Raman spectrometer. The essence of the device (Fig. 5) is a laser source (a solid state laser operating at $785 \mathrm{~nm}$ ), a set of optics based on an Echelle grating and a charge coupled device (CCD) array as a detector. The detector feeds into a computer for data processing and display.

The result is an instrument that produces a plot of scattered intensity as a function of wavenumber with no moving parts. Instead of a microscope and fixed sample chamber, this spectrometer uses fiber optic cable to connect the laser, the sample probe, and the spectrograph. The probe contains a highly efficient narrow band-pass filter that eliminates the need for a second monochromator to discriminate the Raman signal from stray light. The spatial resolution of the probe is less than that of the microscope but has the advantage of being flexible so that spectra can be measured from any object regardless of its size. Further, because the instrument is small, has no moving parts, requires no cooling water for the laser, has modest power requirements, and has no delicate focusing optics, it can be adapted for field use. It would not be impossible to transport the instrument into a cave for in-situ measurements.

The quality of signal obtained from the fiber optic probe spectrograph is illustrated with the spectrum of gypsum (Fig. 6). The bands are sharp and in agreement with single crystal measurements (Berenblut et al., 1971). The intense band at $1008 \mathrm{~cm}^{-1}$ is the symmetric stretching mode of the $\mathrm{SO}_{4}{ }^{2-}$ tetrahedron. Although this band is similar to the $1084 \mathrm{~cm}^{-1}$ band in calcite, the wavenumber shift is significant and represents the distinction between a tetrahedral molecular unit and

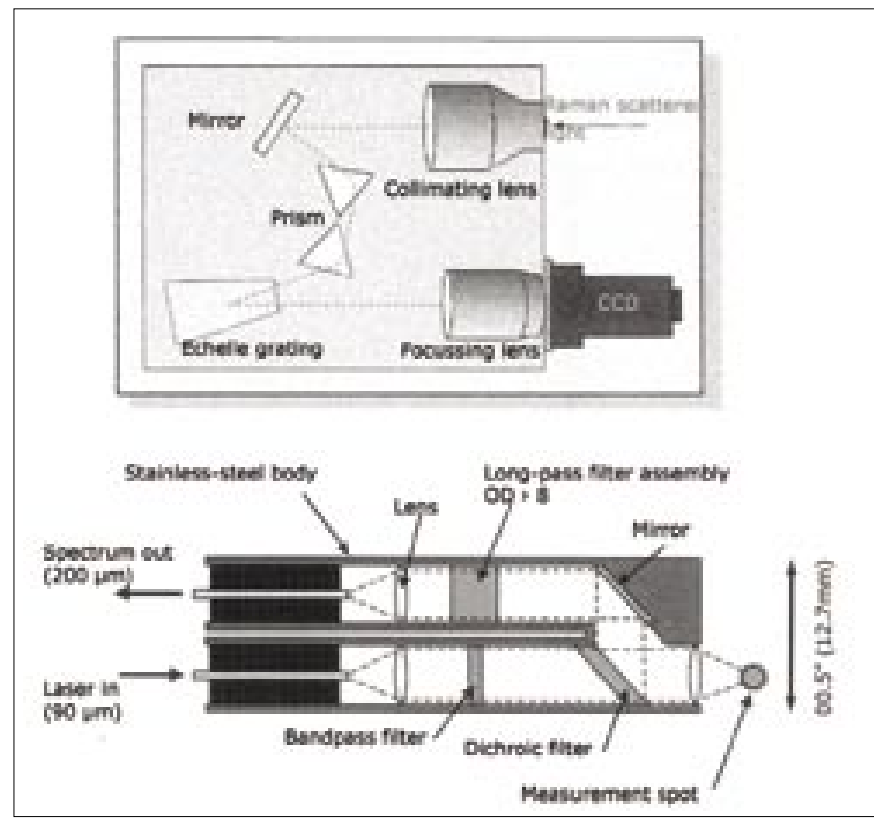

Fig. 5. (A) Spectrograph showing internal optical system. (B) Internal optics of the probe. Not shown are the laser and the optical fibers that connect laser, probe, and spectrograph. Drawings provided by InPhotonics, Inc. a triangular one. The high wavenumber modes are characteristics of the specific molecular anion. One could identify an unknown mineral as a carbonate or a sulfate even in the absence of reference spectra for the identification of the specific mineral. In addition to the sulfate anion, gypsum contains two waters of crystallization. These appear as bands in the very high wavenumber region around $3500 \mathrm{~cm}^{-1}$.

An additional capability of the fiber optic probe is illustrated in Figure 7. Epsomite, $\mathrm{MgSO}_{4} \cdot 7 \mathrm{H}_{2} \mathrm{O}$, is stable in closed containers but tends to dehydrate to hexahydrite, $\mathrm{MgSO}_{4} \cdot 6 \mathrm{H}_{2} \mathrm{O}$ under ambient conditions. The Raman probe has a working distance of $5 \mathrm{~mm}$. It can be focused on a chip of epsomite contained in a glass vial. The broad feature near $1400 \mathrm{~cm}^{-1}$ is related to the glass as confirmed by focusing the probe on the glass itself. The broad bands in the glass spectrum arise because of the disordered structure of glass (Mysen et al., 1982).

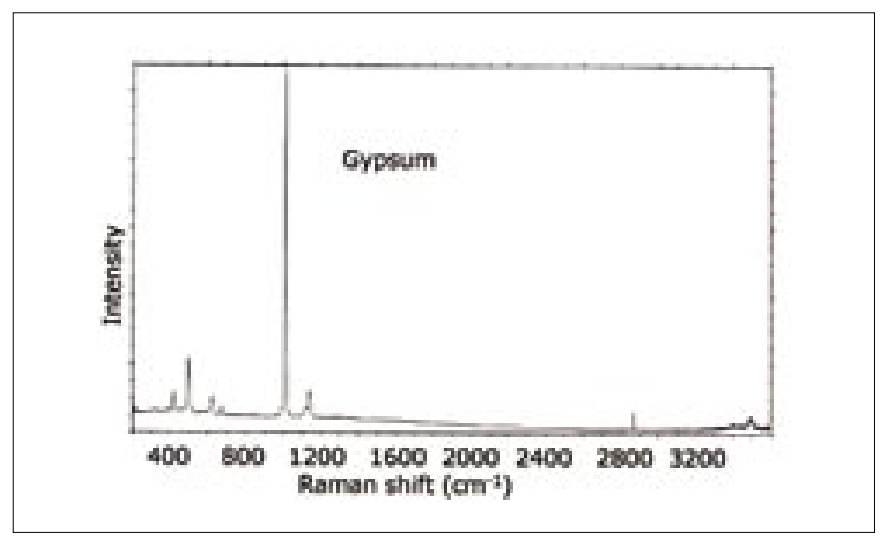

Fig. 6. Raman spectrum of gypsum. Note bands near $3400 \mathrm{~cm}^{-1}$ which are due to the waters of crystallization.

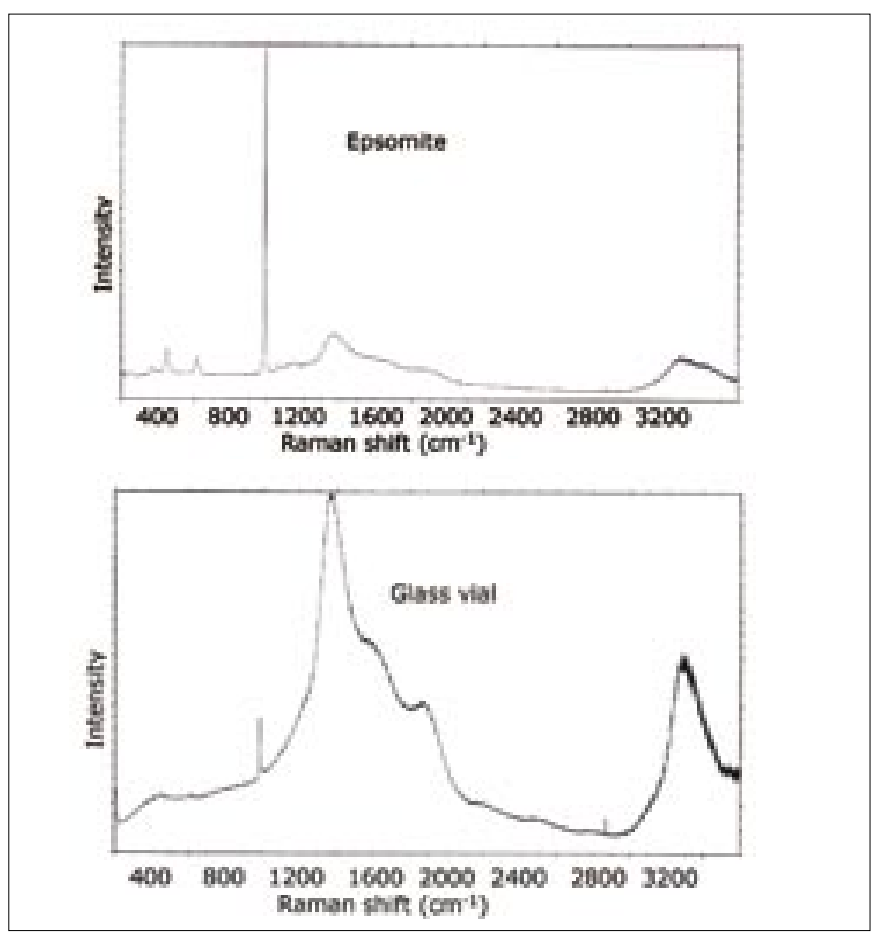

Fig. 7. (A) Raman spectrum of epsomite taken through the glass wall of a shell vial. (B) the spectrum obtained when the probe was focused on the glass. 


\section{CONCLUSIONS}

The purpose of this paper was to call attention to the cave and karst community of new possibilities for the analysis of cave materials. It has been demonstrated that both the microfocus Raman spectrometer and the new fiber-optic probe Raman spectrometer are useful devices for the determination of speleothem mineralogy. Although the probe device has not yet been taken into a cave, it's weight, compactness, lack of need for cooling water, and low power consumption bring in-cave analyses to the threshold of possibility.

The Raman spectrum serves two purposes. First, the pattern of Raman bands can be used purely as a fingerprint. Minerals can be identified by simply matching the observed spectrum against a catalog of reference spectra. If one were to use Raman spectroscopy for a specific objective such as cave mineralogy, it would be a relatively simple task to accumulate a library of reference spectra for the minerals of interest. In this sense, Raman spectra are used in the same way as powder X-ray diffraction patterns. However, the bands of a Raman spectrum relate directly to the bond strengths and atomic masses of the sample. Thus, molecular groups such as the carbonate ion, the sulfate ion, the hydroxyl ion, and water of crystallization can be recognized directly even if no reference spectra are available.

\section{ADDED NOTE}

This paper was originally prepared in early 2005 for presentation at the International Congress of Speleology. The data presented in this paper were obtained with an InPhotonics Model RS2000 instrument. The spectrograph is a benchtop instrument about 0.6 meters in longest dimension and weighing about $20 \mathrm{~kg}$. In addition there is a desktop computer and monitor and a separate small box containing the laser. The instrument is easily moved but not exactly portable. As this writing (December, 2005) B\&W Tek Inc. is advertising a box $22 \mathrm{~cm}$ in longest dimension, weighing about $2 \mathrm{~kg}$, that plugs into a laptop and comes with a carrying handle. Although the author has not had the opportunity to personally examine this instrument, it appears that truly portable Raman spectroscopy has arrived.

\section{REFERENCES}

Adar F., 2001 - Evolution and revolution of Raman instrumentation - Application of available technologies to spectroscopy and microscopy. Chap. 2 In: Lewis I.R. \& Edwards H.G.M. (Eds.), Handbook of Raman Spectroscopy, Marcel Dekker, New York: 11-40.

Berenblut B.J., Dawson P., \& Wilkinson G.R., 1971 The Raman spectrum of gypsum. Spectrochimica Acta, 27A: 1849-1863.

Kiefert L., Hänni H.A. \& Ostertag T., 2001 - Chap. 11 In: Lewis I.R. \& Edwards H.G.M. (Eds.), Handbook of Raman Spectroscopy, Marcel Dekker, New York: 469489.

Knight D.S. \& White W.B., 1989 - Characterization of diamond films by Raman spectroscopy. Journal of Materials Research, 4: 385-393.

Long D.A., 1977 - Raman Spectroscopy. McGraw-Hill, New York. 276 pp.

Mysen B.O., Virgo D. \& Siefert F.A., 1982 - The structure of silicate melts: Implications for chemical and physical properties of natural magmas. Reviews of Geophysics and Space Physics, 20: 353-383.

Smith G.D. \& Clark R.J.H., 2004 - Raman microscopy in archaeological science. Journal of Archaeological Science, 31: 1137-1160.

White W.B., 1974 - The carbonate minerals. Chap. 12. In: Farmer V.C. (Ed.), The Infrared Spectra of Minerals, Mineralogical Society of London: 227-284.

White W.B., 1975 - Structural interpretation of lunar and terrestrial minerals by Raman spectroscopy. Chap. 13. In: Karr Jr., C.C. (Ed.), Infrared and Raman Spectroscopy of Lunar and Terrestrial Minerals, Academic Press, New York. 325-358.

Wilson E.B., Jr., Decius J.C., \& Cross P.C., 1955 Molecular Vibrations: The Theory of Infrared and Raman Vibrational Spectra. McGraw-Hill, New York. 388 pp. 